\title{
Model-independent constraints on cosmic curvature: implication from the future space gravitational-wave antenna DECIGO
}

\author{
Xiaogang Zheng ${ }^{1}$, Shuo $\mathrm{Cao}^{2, \mathrm{a}}$, Yuting Liu ${ }^{2}$, Marek Biesiada ${ }^{2,3}$, Tonghua Liu ${ }^{2,3}$, Shuaibo Geng ${ }^{2}$, Yujie Lian $^{2}$, \\ Wuzheng Guo ${ }^{2}$ \\ ${ }^{1}$ School of Electrical and Electronic Engineering, Wuhan Polytechnic University, Wuhan 430023, China \\ 2 Department of Astronomy, Beijing Normal University, Beijing 100875, China \\ ${ }^{3}$ National Centre for Nuclear Research, Pasteura 7, 02-093 Warsaw, Poland
}

Received: 28 September 2020 / Accepted: 23 December 2020 / Published online: 11 January 2021

(C) The Author(s) 2021

\begin{abstract}
In order to estimate cosmic curvature from cosmological probes like standard candles, one has to measure the luminosity distance $D_{L}(z)$, its derivative with respect to redshift $D_{L}^{\prime}(z)$ and the expansion rate $H(z)$ at the same redshift. In this paper, we study how such idea could be implemented with future generation of space-based DECi-hertz Interferometer Gravitational-wave Observatory (DECIGO), in combination with cosmic chronometers providing cosmology-independent $H(z)$ data. Our results show that for the Hubble diagram of simulated DECIGO data acting as a new type of standard siren, it would be able to constrain cosmic curvature with the precision of $\Delta \Omega_{k}=0.09$ with the currently available sample of 31 measurements of Hubble parameters. In the framework of the third generation ground-based gravitational wave detectors, the spatial curvature is constrained to be $\Delta \Omega_{k}=0.13$ for Einstein Telescope (ET). More interestingly, compared to other approaches aiming for model-independent estimations of spatial curvature, our analysis also achieve the reconstruction of the evolution of $\Omega_{k}(z)$, in the framework of a model-independent method of Gaussian processes (GP) without assuming a specific form. Therefore, one can expect that the newly emerged gravitational wave astronomy can become useful in local measurements of cosmic curvature using distant sources.
\end{abstract}

\section{Introduction}

The spatial curvature parameter $\Omega_{k}$ is a very significant quantity closely related to many fundamental issues in modern cosmology, such as the structure and evolution of the Universe $[1,2]$. The most popular concept of the very early Universe undergoing an exponential phase of expansion pre-

a e-mail: caoshuo@bnu.edu.cn (corresponding author) dicts that the radius of curvature of the Universe should be very large, which means that cosmic curvature should be close to zero [3]. Current cosmological observations, e.g., the combined Planck 2018 cosmic microwave background (CMB) and baryon acoustic oscillation (BAO) measurements, strongly favor this inflation theory and demonstrate the flatness of the Universe $\left(\Omega_{k}=0.001 \pm 0.002\right)$ [4]. However, one should note that such stringent constraint on the cosmic curvature is indirect and strongly relying on the pre-assumption of a specific cosmological model (i.e., the cosmological constant plus cold dark matter model, usually abbreviated as $\Lambda \mathrm{CDM}$ model). In general, most studies focusing on the cosmic curvature always assume that dark energy is just a cosmological constant, while the Universe is assumed flat in most of the dark energy studies. However, recent analysis indicated that cosmological constant assumption might cause tension between $\Lambda \mathrm{CDM}$ and dynamical dark-energy model, while flat Universe assumption may lead to an incorrect reconstruction of the dark energy equation of state [5-10]. Besides, the combination of the Planck 2018 $T T, T E, E E+$ low $E$ power spectra data alone slightly favor a mildly closed Universe, i.e., $\Omega_{k}=-0.044_{-0.015}^{+0.018}[4,11]$. Any small change in the spatial curvature could have a huge impact on the reconstructed history of the Universe. Therefore, purely geometrical and model-independent methods of inferring the spatial curvature have always been an important issue in cosmology.

In particular, the distance sum rule [12], which characterizes the relation between the distances of the background source, the lens and the observer in the Friedmann-LemaitreRobertson-Walker (FLRW) metric, has been proposed as a model-independent method to constrain the curvature of the Universe [13]. Such methodology was then applied to test the validity to FLRW metric, based on the galactic-scale lensing systems where strongly lensed gravitational waves and their 
electromagnetic counterparts can be simultaneously detected [10]. More recently, Qi et al. [14], Zhou et al. [15] extended the cosmic curvature analysis to higher redshift, using the latest data sets of strong lensing systems $[16,17]$ combined with intermediate-luminosity quasars calibrated as standard rulers [18]. Another straightforward method to constrain the cosmic curvature has been proposed by Clarkson et al. [6], using the expansion rate measurements $\mathrm{H}(\mathrm{z})$ and the transverse comoving distances $D(z)$. Such method is also model-independent, which has been further developed with updated observational SNe Ia data acing as standard candles [19-24,26-28], ultracompact structures in radio quasars acting as standard rulers [10], and the Hubble diagram based on the non-linear relation between the UV and X-ray monochromatic luminosities of quasars [29-31].

As a new window on the Universe, gravitational wave (GW) signals create alternative opportunities. Namely, the GW signals from inspiralling binary black holes $(\mathrm{BH}-\mathrm{BH})$ and neutron stars (NS-NS) (or mixed BH-NS systems) can be used as standard sirens providing the luminosity distances in a direct way, not relying on the cosmic distance ladder [32]. Compared with the observations of SNe Ia in the electromagnetic (EM) domain, the greatest advantage of GWs is the independent calibration of luminosity distances. Some recent studies have discussed the possibility of extending the cosmic curvature test based on the simulated data of GW from future gravitational wave detectors [33-35]. In this paper, we will consider the reconstruction of cosmic curvature parameter $\Omega_{k}$ at different redshifts [6], focusing on the standard sirens accessible to the future space-borne GW detector, i.e., the DECi-hertz Interferometer Gravitational wave Observatory (DECIGO), as well as the third generation GW groundbased detector, i.e., the Einstein Telescope (ET). In the EM domain, the recent measurements of Hubble parameters $H(z)$ are inferred from the differential ages of galaxies, i.e., cosmic chronometers (CC) acting as standard clocks. In order to investigate the redshift dependence of $\Omega_{k}$ without assuming any specific functional form, the so-called Gaussian processes (GP) [36] will also be applied to reconstruct the evolution of the curvature of the universe, which has been widely used in recent works [37-41]. This paper is organized as follows. In Sect. 2, we give a brief introduction of the methodology and data used in this work. Our results and discussions are presented in Sect. 3. Finally, the general conclusions are summarized in Sect. 4.

\section{Methodology and observational data}

Under the assumption that the Universe is homogeneous and isotropic on the large scales, the FLRW metric can be used to describe its geometry as $d s^{2}=-d t^{2}+a^{2}(t)\left[\frac{d r^{2}}{1-K r^{2}}+r^{2}\left(d \theta^{2}+\sin ^{2} \theta d \phi^{2}\right)\right]$,

where $t$ is the cosmic time and $(r, \theta, \phi)$ are the comoving spatial coordinates. The scale factor $a(t)$ is the only gravitational degree of freedom and its evolution (by virtue of the Einstein's equations) is determined by the matter and energy of the Universe. The dimensionless curvature $K=-1,0,+1$ corresponds to open, flat and closed Universe, respectively. In such metric, the luminosity distance $D_{L}(z)$ can be expressed as

$$
D_{L}(z)= \begin{cases}\frac{c(1+z)}{H_{0} \sqrt{\left|\Omega_{\mathrm{k}}\right|}} \sinh \left[\sqrt{\left|\Omega_{\mathrm{k}}\right|} \int_{0}^{z} \frac{d z^{\prime}}{E\left(z^{\prime}\right)}\right] & \text { for } \Omega_{K}>0 \\ \frac{c(1+z)}{H_{0}} \int_{0}^{z} \frac{d z^{\prime}}{E\left(z^{\prime}\right)} & \text { for } \Omega_{K}=0 \\ \frac{c(1+z)}{H_{0} \sqrt{\left|\Omega_{\mathrm{k}}\right|}} \sin \left[\sqrt{\left|\Omega_{\mathrm{k}}\right|} \int_{0}^{z} \frac{d z^{\prime}}{E\left(z^{\prime}\right)}\right] & \text { for } \Omega_{K}<0 .\end{cases}
$$

The dimensionless Hubble parameter $E(z)$ is defined as $H(z) / H_{0}$, where $H(z)$ is the Universe expansion rate and $H_{0}$ is the Hubble constant. The curvature parameter $\Omega_{k}$ is related to $K$ as $\Omega_{k}=-c^{2} K /\left(a_{0} H_{0}\right)^{2}$, where $c$ is the speed of light. The derivative of Eq. (2) will provide the cosmic curvature $\Omega_{k}$, expressed by the Universe expansion rate $H(z)$ and transverse comoving distance $D(z)$ as [6]

$\Omega_{k}=\frac{\left[H(z) D^{\prime}(z)\right]^{2}-c^{2}}{\left[H_{0} D(z)\right]^{2}}$.

The luminosity distance $D_{L}(z)$ is simply related to the transverse comoving distance $D(z)$ as $D(z)=D_{L}(z) /(1+z)$ [42], while $D^{\prime}(z)=d D(z) / d z$ denotes the derivative with respect to redshift $z$. In our analysis, we use the luminosity distance as a function of redshift $z$ based on simulated standard siren data from DECIGO and ET to reconstruct $D(z)$ and $D^{\prime}(z)$, independently, and combine these two reconstructions with independent $H(z)$ measurements to derive $\Omega_{k}$.

\subsection{Distance from GW standard siren DECIGO and ET}

DECi-hertz Interferometer Gravitational-wave Observatory (DECIGO) project proposed by Japan $[43,44]$ is a future space gravitational-wave antenna, whose currently most attractive science objective is detecting the gravitational waves from the inflation [45]. Unlike the ground-based GW detectors (such as aLIGO and VIRGO), DECIGO was designed most sensitive between 0.1 and $10 \mathrm{~Hz}$ to reach lower frequency detection with drag-free spacecrafts. More importantly, this frequency range fills the gap between sensitivity windows of ground based detectors and future LISA spaceborne detector. In particular, this opportunity would allow the early detection (and measurements) of inspiralling sources which would enter the ground-based detector a few years 
after. This would create unprecedented opportunity to precisely measure properties of such sources and to improve the determination of their position on the sky. Aiming to improve the detection sensitivity, DECIGO implements four clusters of spacecraft, and each cluster consists of three spacecraft with three Fabry-Perot Michelson interferometers, whose arm length is $1000 \mathrm{~km}$. The expected sensitivity is $10^{-25}$ $\mathrm{Hz}^{-1 / 2}$ for two clusters of DECIGO at the same position for three years of mission [46]. Without any pre-assumptions concerning cosmology, signals from inspiraling binary neutron stars (NS-NS) and black holes (BH-BH) can provide an absolute measurement of the luminosity distance $[47,48]$. The GW amplitude is related to the so-called chirp mass (which can be measured from the GW signal's phasing) and the luminosity distance. Therefore, the luminosity distance can be extracted from the amplitude and the rate of frequency change. DECIGO will be able to detect the gravitational waves from neutron star binaries even at a redshift of $z \sim 5$ for five years of its mission and many intermediate-mass black hole binary coalescences.

Focusing on the GW signals from the binary system with component masses $m_{1}$ and $m_{2}$, one can define the chrip mass $M_{c}=M \eta^{3 / 5}$, where $M$ is the total mass of binary system $M=m_{1}+m_{2}$ and $\eta$ is symmetric mass ratio $\eta=m_{1} m_{2} / M^{2}$. In GW experiments, the Fourier transform of time domain waveform can be computed as

$\tilde{h}(f)=\frac{A}{D_{L}(z)} M_{z}^{5 / 6} f^{-7 / 6} e^{i \Phi(f)}$,

where $A$ is a geometrical average over the inclination angle of the binary system $A=\left(\sqrt{6} \pi^{2 / 3}\right)^{-1}, M_{z}$ - the so called redshifted chirp mass, is defined as $M_{z}=(1+z) M_{c}$ and $D_{L}(z)$ is the luminosity distance. $\Phi(f)$ is the frequency-dependent phase caused by orbital evolution which is usually described by post-Newtonian (PN) approximation (an approximation to General Relativity in the weak-field, slow-motion regime) of different order [49,50].

In order to estimate the uncertainty of the measurement of the luminosity distance, one can use the following Fisher matrix

$\Gamma_{a b}=4 \operatorname{Re} \int_{f_{\min }}^{f_{\max }} \frac{\partial_{a} \widetilde{h}_{i}^{*}(f) \partial_{b} \widetilde{h}_{i}(f)}{S_{h}(f)} d f$

where $\partial_{a}\left(\partial_{b}\right)$ is derivative with respect to parameter $\theta_{a}\left(\theta_{b}\right)$. The noise power spectrum $S_{h}(f)$ consists of the shot noise, the radiation pressure noise and the acceleration noise. Based on the mechanical parameters of the DECIGO tuned to the $0.1-10 \mathrm{~Hz}$ frequency window, i.e., the arm length $1000 \mathrm{~km}$, the output laser power $10 \mathrm{~W}$ with wavelength $\lambda=532 \mathrm{~nm}$, the mirror diameter $1 \mathrm{~m}$ with its mass $100 \mathrm{~kg}$, and the finesse of Fabry-Perot Michelson interferometer cavity 10, the noise power spectrum is fitted as [51]

$$
\begin{aligned}
S_{h}(f)= & 6.53 \times 10^{-49}\left[1+\left(\frac{f}{7.36 \mathrm{~Hz}}\right)^{2}\right] \\
& +4.45 \times 10^{-51} \times\left(\frac{f}{1 \mathrm{~Hz}}\right)^{-4} \times \frac{1}{1+\left(\frac{f}{7.36 \mathrm{~Hz}}\right)^{2}} \\
& +4.94 \times 10^{-52} \times\left(\frac{f}{1 \mathrm{~Hz}}\right)^{-4} \mathrm{~Hz}^{-1}
\end{aligned}
$$

For the convenience of calculations, we assume equal mass NS-NS binary system with $m_{1}=m_{2}=1.4 M_{\odot}$. Then we have $M_{z}=1.22(1+z) M_{\odot}$ and $\eta=0.25$. Therefore, the luminosity distance is independent on other parameters in the Fisher matrix and the instrumental uncertainty of the measurement of the luminosity distance can be estimated as

$\sigma_{D_{L}}^{i n s t r}=\sqrt{\Gamma_{a a}^{-1}}$.

Concerning the uncertainty budget, the luminosity distance precision per GW is taken as [52]

$\sigma_{D_{L}}=\sqrt{\left(\sigma_{D_{L}}^{\text {instr }}\right)^{2}+\left(\sigma_{D_{L}}^{\text {lens }}\right)^{2}}$.

In our simplified case, $\sigma_{D_{L}}^{\text {instr }} \simeq \frac{2 D_{L}}{\rho}$, where $\rho$ denotes the signal-to-noise ratio (SNR) of DECIGO interferometers [53]. Meanwhile, the lensing uncertainty caused by the weak lensing can be estimated as $\sigma_{D_{L}}^{\text {lens }}=0.05 z D_{L}$ [54]. As a result, the total uncertainty of $D_{L}$ is modeled as

$\sigma_{D_{L}}=\sqrt{\left(\frac{2 D_{L}}{\rho}\right)^{2}+\left(0.05 z D_{L}\right)^{2}}$

Finally, we employ the redshift distribution of the GW sources observed on Earth expressed as [54]

$P(z) \propto \frac{4 \pi D_{c}^{2}(z) R(z)}{H(z)(1+z)}$,

where $D_{c}(z)$ is comoving distance, $H(z)$ the expansion rate. $R(z)$ is the NS-NS coalescence rate, which according to [55, $56]$ can be approximated as:

$R(z)= \begin{cases}1+2 z, & z \leq 1 \\ \frac{3}{4}(5-z), & 1<z<5 \\ 0, & z \geq 5 .\end{cases}$

In our simulation, we assume the flat $\Lambda \mathrm{CDM}$ as our fiducial cosmological model with the matter density parameter and the Hubble constant values $\left(\Omega_{m}=0.315, H_{0}=\right.$ $67.4 \mathrm{~km} \mathrm{~s}^{-1} \mathrm{Mpc}^{-1}$ ) taken after Planck CMB measurements [4]. Recent analysis of [46] suggests that the space-based GW detector DECIGO can detect up to $10,000 \mathrm{GW}$ events up to redshift $z \sim 5$ in one year of operation. Thus, we simulate a mock data of $10,000 \mathrm{GW}$ events to be used for the cosmic curvature constraint analysis. The redshift distribution of our mock catalog is shown in Fig. 1. In order to 


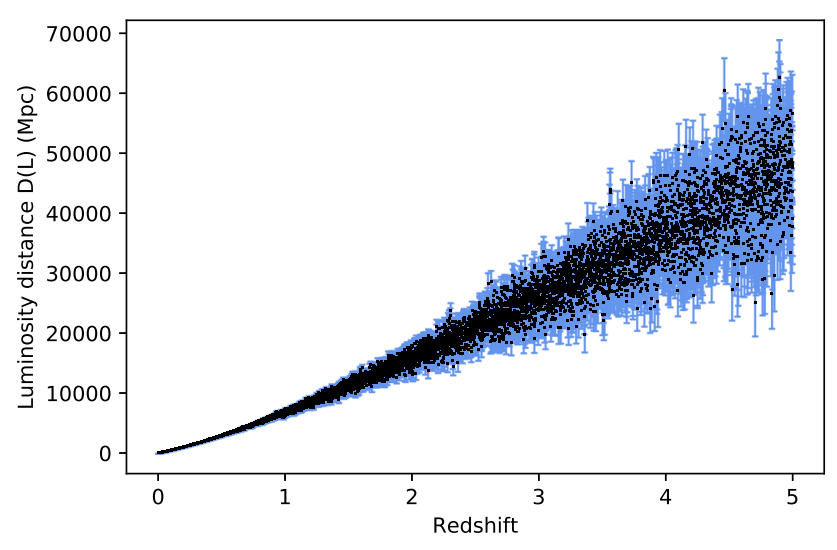

Fig. 1 The scatter plot of the luminosity distances in the catalog of 10,000 simulated GW events detectable by the DECIGO

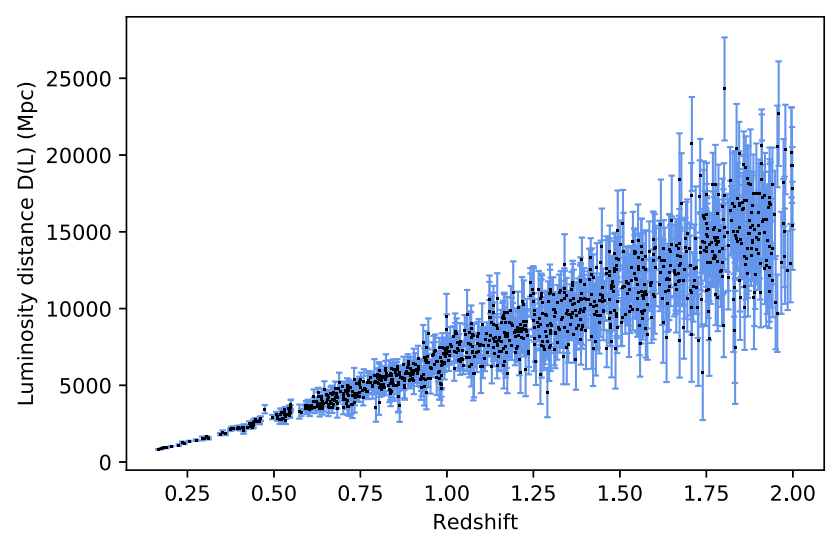

Fig. 2 The scatter plot of the luminosity distances in the catalog of 1000 simulated GW events observable by the third generation detector ET

improve the reconstruction of $D(z)$ we also simulate $\mathrm{GW}$ events observable by the Einstein Telescope (ET), which is the planned third-generation ground-based GW detector [57]. Compared to the current advanced ground-based detectors, ET was designed more sensitive between $1 \mathrm{~Hz}$ and $10^{4}$ Hz. This and increased overall sensitivity, will expand the detection space by three orders of magnitude [57]. As proposed by the design document, it is made up of three collocated underground detectors, each with $10 \mathrm{~km}$ arm and with a $60^{\circ}$ opening angle. Theoretically, ET will be able to detect GWs from the NS-NS mergers up to the redshift $z \sim 2$ and BH-NS mergers up to the redshift of $z \sim 5$ [52]. In our analysis, we perform the Monte Carlo simulation of ET detectable GW signals from NS-NS systems up to $z \sim 2$. Specific simulation steps are similar to those used in $[58,59]$. The mock catalog of 1000 simulated GW events observable by the ET is displayed in Fig. 2.

Our goal is to employ Eq. (3) for the assessment of cosmic curvature. For this purpose we need not only $D_{L}(z)$ but also the derivative of this function. Such derivative can be reconstructed by many different methods. We have chosen the following approach. First, we make an empirical fit to the luminosity distance based on a third-order logarithmic polynomial as in as in Risaliti \& Lusso [29]

$D_{L}^{P}(z)=\frac{c}{H_{0}} \ln (10)\left[x+a_{2} x^{2}+a_{3} x^{3}+\mathcal{O}\left(x^{4}\right)\right]$

where $x=\log (1+z), a_{2}$ and $a_{3}$ are two constants that need to be fitted to the standard siren data sets. Compared to other empirical fitting methods, such logarithmic parametrization has an advantage of faster convergence at higher redshifts $(0<z<0)$. Let us remark that cosmography based on the logarithmic polynomial expansion is expected to break down at $z>2$ and produce spurious effects as discussed in [70]. We will discuss it further in the next section. For now, let us stress that in the redshift range of $H(z)$ on which we fit our cosmographic formula 12 the convergence is reasonable. More specifically, to obtain the best-fit values of parameters and their uncertainties, we use the Markov Chain Monte Carlo (MCMC) method introduced by Foreman-Mackey et al. [60] and implemented in the Python module called emcee. ${ }^{1}$ For the standard siren data sets, the parameters $a_{2}$ and $a_{3}$ characterizing the luminosity distance are optimized by minimizing the $\chi^{2}$ objective function

$\chi^{2}=\sum_{i=1}^{n} \frac{\left[D_{L}^{G W}-D_{L}^{P}\right]^{2}}{\sigma_{G W}^{2}}$,

where $\sigma_{G W}^{2}$ can be obtained from the Eq. (10). The marginalized probability distribution of each parameter and the corresponding marginalized 2-D confidence contours are presented in Figs. 3 and 4.

\subsection{The expansion rate measurements $H(z)$}

The expansion rate (i.e., the Hubble parameter $H(z))$ measurements have been widely used in the research of the nature of dark energy and the evolution of the Universe. The idea of using the differential age evolution of passively evolving galaxies has been proposed by Jimenez et al. [61] as a method independent on any assumptions regarding the details of the cosmological model. Namely, one can write the definition of the expansion rate in the following form:

$H(z)=\frac{\dot{a}}{a}=-\frac{1}{1+z} \frac{d z}{d t}$.

The derivative of the redshift $z$ with respect to cosmic time $t$ can be directly obtained by measuring the age difference between the two galaxies at different redshifts. In the case of early-type galaxies evolving passively on a timescale longer than their age difference, certain features of their spectra, such as the D4000 break enable us to measure the age difference of such galaxies. This is so-called differential age (DA)

\footnotetext{
${ }^{1}$ https://pypi.python.org/pypi/emcee.
} 


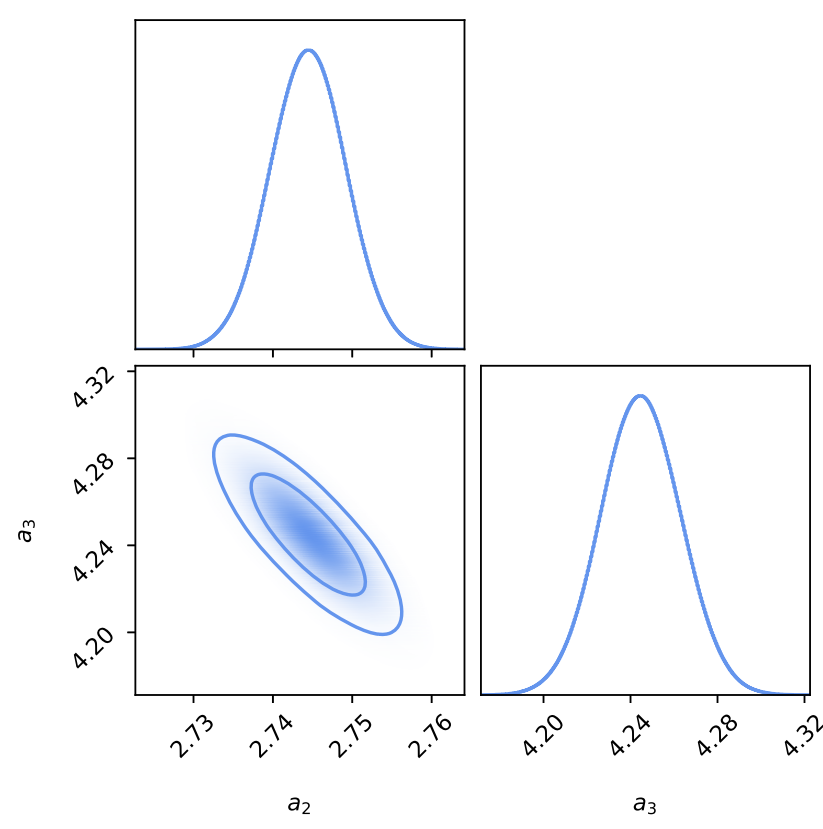

Fig. 3 Marginalized constraints on $a_{2}$ and $a_{3}$ based on the standard sirens catalog from the DECIGO

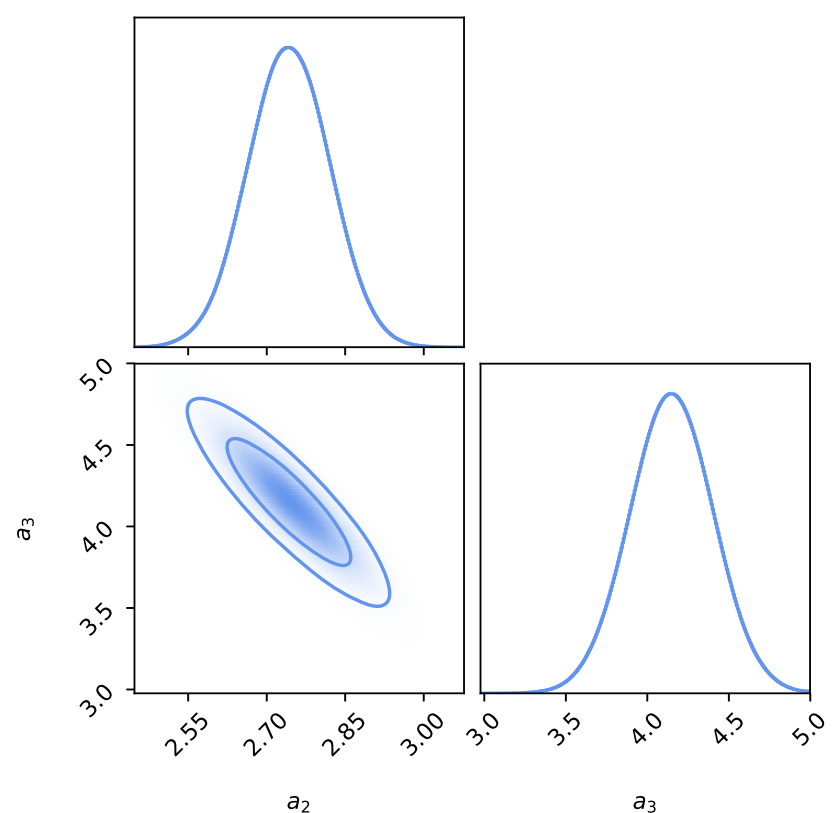

Fig. 4 Marginalized constraints on $a_{2}$ and $a_{3}$ based on the standard sirens catalog from the third generation GW detector ET

or cosmic chronometer (CC) approach. There is also another approach to obtain the $H(z)$ data based on the radial baryon acoustic oscillations (BAO) features from galaxy clustering [62-66]. However, the $H(z)$ data obtained employing such method are based on an assumed fiducial cosmological model and the prior for the distance to the last scattering surface from CMB observations [24]. Moreover, there exist a deviation between those two $H(z)$ compilations [25]. There- fore, we do not use $H(z)$ data obtained from BAO. Consequently, we use only the latest $\mathrm{CC}$ measurements of $H(z)$ comprising totally 31 data-points covering the redshift range $0.070<z<1.965$.

Besides using only a small sample of available $H(z)$ data to assess the cosmic curvature we also reconstruct the $H(z)$ function from the data. For this purpose we employ a smoothing technique based on Gaussian processes (GP). Such an approach, firstly proposed by [36], has been extensively applied in various studies [22,41,67-69]. The crucial issue in the GP technique is to determine the covariance function, with which one can derive the quantities at the redshifts, where they have not been directly measured. In this work, we use the squared exponential covariance function

$k(x, \tilde{x})=\sigma_{f}^{2} \exp \left(-\frac{(x-\tilde{x})^{2}}{2 \ell^{2}}\right)$,

where $x$ and $\tilde{x}$ are any two different points, $\ell$ and $\sigma_{f}$ are two hyperparameters, which characterize the bumpiness of the function and can be optimized by GP with the observed data set. $\ell$ can be thought of as the characteristic length one has to travel in $x$-direction to get a significant change in $f(x)$, whereas $\sigma_{f}$ denotes the typical change in the y-direction. In contrast to the Matérn and Cauchy covariance function, which are another popular choices, the squared exponential covariance function has the advantage that it is infinitely differentiable, which is useful for reconstructing the derivative of the function [36,41]. By using the squared exponential covariance function and the zero mean function $\mu(z)$ (another ingredient of GP approach), we can obtain the values of data points at other redshifts which have not be observed. This can effectively bridge the redshift gaps between current data-points. Technically, we use the code called GaPP in Python $^{2}$ to derive our GP results. The latest measurements of 31 Hubble parameters obtained from CCs and the resulting GP reconstruction of $H(z)$ function are presented in Fig. 5.

Now, we combine the reconstructed $D(z)$ and $D^{\prime}(z)$ with the Hubble parameter $H(z)$ to constrain the $\Omega_{k}(z)$ according to Eq. (3). Let us stress again that the standard sirens and cosmic chronometers are independent of each other, which indicates that such determination of the cosmic curvature is model-independent.

\section{Results and discussion}

By applying the above described procedure to $D_{L}(z)$ reconstructed from simulated catalog of standard sirens observable by DECIGO and $H(z)$ data from cosmic chronometers, we obtain 31 independent measurements of the cosmic curvature $\Omega_{k}(z)$ at each redshift $z$, where $H(z)$ is measured. They

\footnotetext{
${ }^{2}$ https://github.com/carlosandrepaes/GaPP.
} 


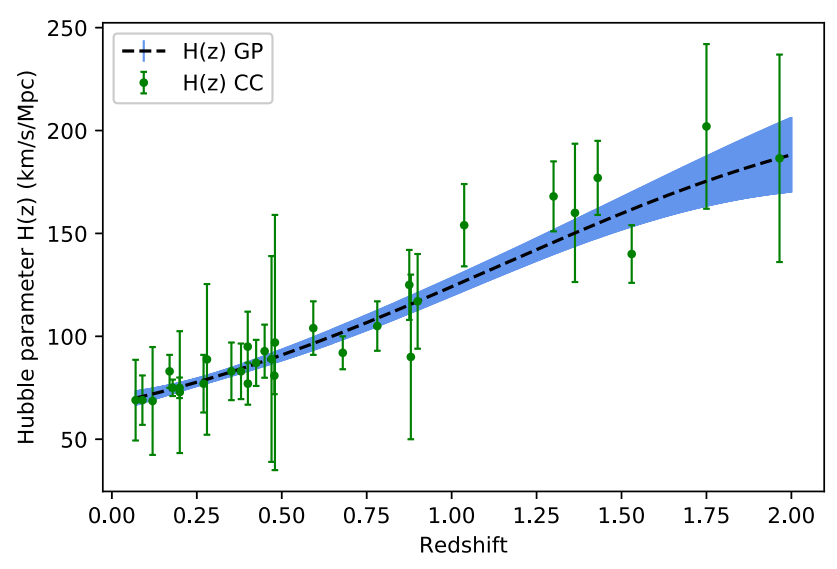

Fig. 5 The latest measurements of 31 Hubble parameters from the galaxy differential age method and the $H(z)$ reconstruction function with GP. Green dots denote CC measurements with corresponding 1- $\sigma$ uncertainties. Black line and blue region represent the reconstructed $H(z)$ function and its $1-\sigma$ uncertainty band

are shown in Fig. 6. The uncertainty bars displayed on this figure were calculated from the uncertainty budget of $D(z)$, $D^{\prime}(z)$ and $H(z)$.

One can summarize these measurements with the inverse variance weighted mean

$$
\begin{aligned}
\bar{\Omega}_{k} & =\frac{\sum\left(\Omega_{k, i} / \sigma_{\Omega_{k, i}}^{2}\right)}{\sum 1 / \sigma_{\Omega_{k, i}}^{2}}, \\
\sigma_{\bar{\Omega}_{k}}^{2} & =\frac{1}{\sum 1 / \sigma_{\Omega_{k, i}}^{2}},
\end{aligned}
$$

where $\bar{\Omega}_{k}$ stands for the weighted mean of cosmic curvature and $\sigma_{\bar{\Omega}_{k}}$ is its uncertainty. We find that, combination of future DECIGO standard sirens and currently available CC data gives $\bar{\Omega}_{k}=0.004 \pm 0.09$. It is fully consistent with the vanishing curvature - an assumption underlying our GW data simulations and compatible with the constraints obtained from the latest Planck CMB measurements [4]. Let us recall that we are combining simulated and real data. The precision of this estimate is comparable to the recent analysis of Cao et al. [10], which represented the precision of $\Delta \Omega_{k} \sim 10^{-2}$ with 250 well-observed radio quasars. Such conclusion is also well consistent with the cosmic curvature derived from the strong lensing and supernova distance measurements in the framework of another model-independent test based on the distance sum rule [15].

When the current $\mathrm{CC}$ data are combined with forecasts for the ET ground-based detector, one obtains $\bar{\Omega}_{k}=0.009 \pm$ 0.13. Individual reconstructed $\Omega_{k}(z)$ are shown in Fig. 7. Again the results are compatible with vanishing cosmic curvature, however the precision of the final result is much worse.

Since our simulated GW catalogs used to derive $D_{(z)}$ and $D_{L}^{\prime}(z)$ are rich enough we attempted at dividing the full sample into different redshift bins and assessed $\bar{\Omega}_{k}$ in

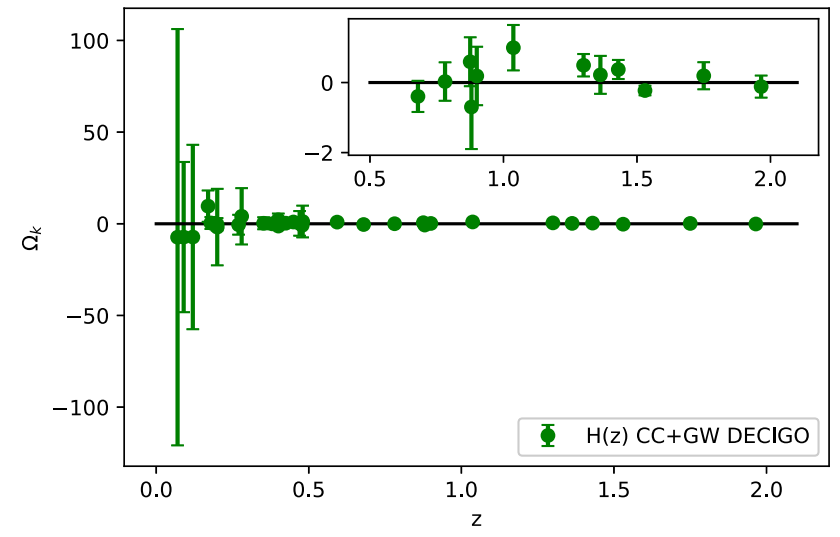

Fig. 631 measurements of the cosmic curvature parameter $\Omega_{k}$ from the standard sirens of GW space-based detector DECIGO and cosmic chronometers. Inset in the upper right reveals more details

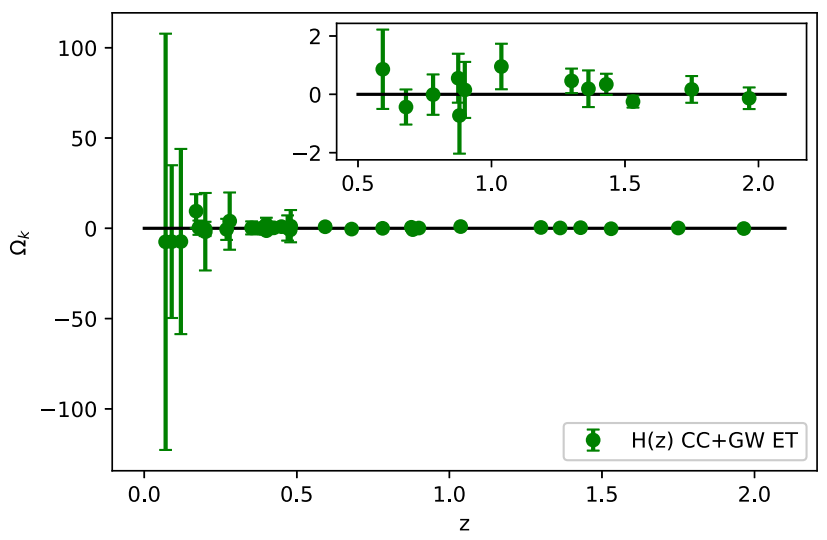

Fig. 731 measurements of the cosmic curvature parameter $\Omega_{k}$ from the standard sirens of GW third-generation ground-based detector ET and cosmic chronometers. Inset in the upper right reveals more details

Table 1 Weighted average of $\Omega_{k}$ in different redshift bins from the standard siren of GW detector (DECIGO and ET) and standard clock of cosmic chronometers $\mathrm{H}(\mathrm{z})$

\begin{tabular}{lll}
\hline Redshift bins & $C C+D E C I G O$ & $C C+E T$ \\
\hline $0-2.0$ & $0.004 \pm 0.09$ & $0.009 \pm 0.13$ \\
$0-0.5$ & $-0.22 \pm 0.63$ & $-0.23 \pm 0.74$ \\
$0.5-1.0$ & $-0.02 \pm 0.27$ & $-0.03 \pm 0.34$ \\
$1.0-1.5$ & $0.45 \pm 0.19$ & $0.41 \pm 0.24$ \\
$1.5-2.0$ & $-0.17 \pm 0.12$ & $-0.17 \pm 0.17$ \\
\hline
\end{tabular}

each bin. More specific, we divide the $H(z)$ measurements into four groups, including 18 data points with redshifts $z<0.5,6$ data points with redshifts $0.5<z<1.0,4$ data points with redshifts $1.0<z<1.5$ and 3 data points with $1.5<z<2.0$. The cosmic curvature parameter obtained in these sub-samples is presented in Table 1. Note that the derived curvature is negative when $z>1.5$, which is also 
Table 2 Weighted average of $\Omega_{k}$ in different redshift bins from $\mathrm{CC}+\mathrm{DECIGO}$ based on fourth and fifth order logarithmic polynomial

\begin{tabular}{lll}
\hline Redshift bins & 4th-order polynomial & 5th-order polynomial \\
\hline $0-2.0$ & $0.01 \pm 0.10$ & $0.0001 \pm 0.11$ \\
$0-0.5$ & $-0.29 \pm 0.66$ & $-0.25 \pm 0.66$ \\
$0.5-1.0$ & $-0.06 \pm 0.29$ & $-0.07 \pm 0.28$ \\
$1.0-1.5$ & $0.46 \pm 0.20$ & $0.42 \pm 0.22$ \\
$1.5-2.0$ & $-0.15 \pm 0.13$ & $-0.16 \pm 0.15$ \\
\hline
\end{tabular}

consistent with the results from the model-dependent constraints in the literature [22].

Finally, as mentioned in [70] the third-order logarithmic polynomial cannot be trusted at high redshift range (especially $z>2$ region) and the influence of the breakdown of the validity of log-polynomial expansion was discussed in that paper. In order to check the performance of our approach based on the third order expansion and to see how it relates to the findings of [70], we repeated the whole procedure with 4th and 5th-order logarithmic polynomial expansions for different redshift bins. Corresponding results are listed in Table 2. It can be seen that next orders of expansion give the results compatible to the third order one. Of course, the order at which we truncate the logarithmic polynomial expansion affects the final result, but up to $z \approx 2$ the deviations are not dramatic, which is consistent with previous investigations [70,71].

Moreover, we performed the $\Omega_{k}(z)$ reconstruction using not the point (or binned) CC data but the $H(z)$ reconstruction using a model-independent smoothing technique of Gaussian processes [36]. Figure 8 displays such reconstructed $\Omega_{k}$ parameter as a function of redshift for two different scenarios: combined CC + DECIGO data and CC + ET data. We expect that as the precision of the future data improves (especially the Hubble parameter $H(z)$ based on the full sky BAO survey up to redshift $z \sim 5$ [3]), our approach will yield an even more accurate determination of $\Omega_{k}$, especially at higher redshifts [38]. We also repeated the $\Omega_{k}$ reconstruction with cosmography based on 4th and 5th-order log polynomial expansions. The results turned out indistinguishable from those shown in Fig. 8. Certainly, the impact of the breakdown of polynomial expansions should be investigated before any cosmological applications especially involving data at high redshifts. This would be particularly important in the precision cosmology era.

\section{Conclusions}

In this paper, we applied a model-independent technique to constrain the cosmic curvature parameter $\Omega_{k}$ at different redshift points directly, based on the idea expressed by Clarkson
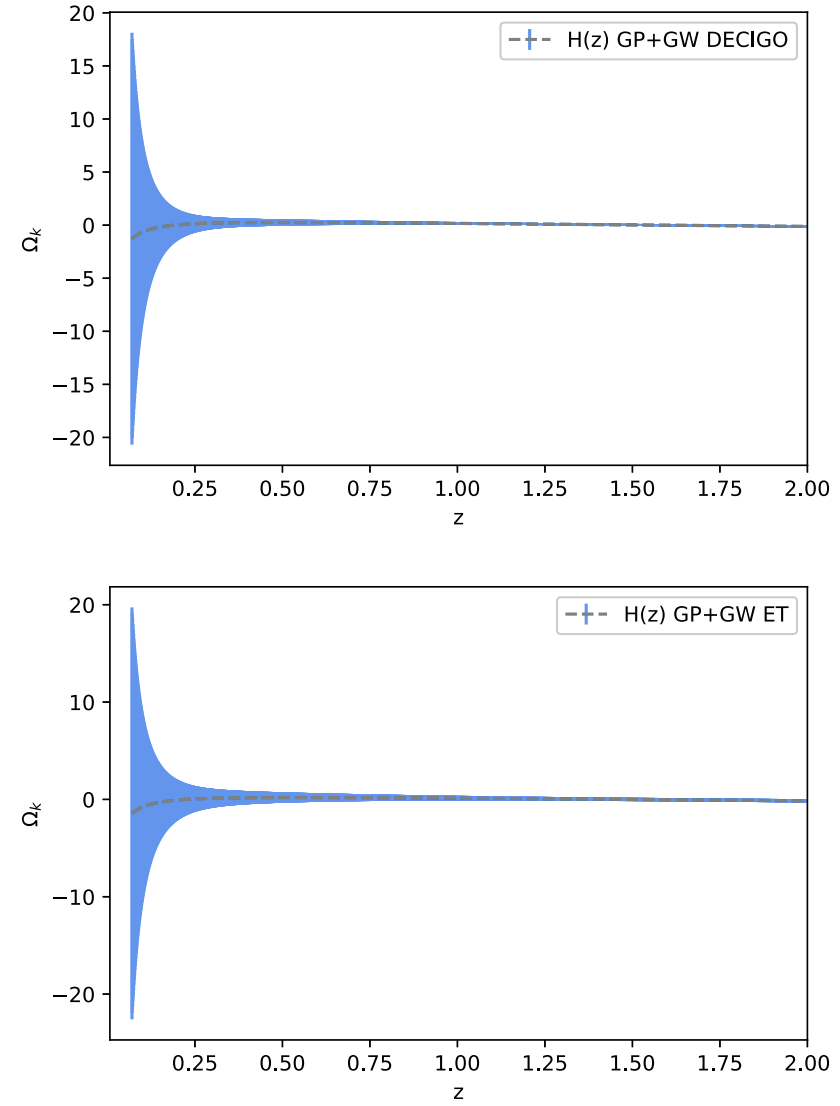

Fig. 8 Gaussian process reconstruction of $\Omega_{k}$ obtained from the combined data sets of $H(z)+D E C I G O$ (upper) and $H(z)+E T$ (lower). The blue regions are the $68 \%$ C.L. of the reconstructions

et al. [6]. Because currently we do not have samples of standard candles $\left(D_{L}(z)\right.$ measurements) rich enough to implement this method, we used simulations of standard sirens data obtainable from DECIGO and ET future space-borne and ground-based detectors. They are expected to register $\mathcal{O}\left(10^{4}\right)$ NS-NS inspirals up to $z \sim 5$ or $\mathcal{O}\left(10^{4}\right)$ such events up to $z \sim 2$, respectively. The second necessary ingredient of this method are independently obtained measurements of expansion rates at different redshift. For this purpose we used the current data obtained from passively evolving galaxies (CC) covering the redshift range of $0.07<z<2$.

Firstly, from simulated $D_{L}$ samples representing future standard siren observations (DECIGO and ET) we reconstructed the transverse comoving distance $D(z)$ and its derivative $D^{\prime}(z)$ with respect to redshift $z$. In order to achieve this goal not relying on any specific cosmological model, we used the third-order logarithm polynomial approximation of $D_{L}(z)$ with undetermined coefficients, which could be optimized numerically. Then, one was able to directly calculate the curvature parameter $\Omega_{k}$ combining these results with the expansion rate $H(z)$ measurements obtained from a sample of cosmic chronometers. 
We found that the cosmic curvature could be constrained as $\Omega_{k}=0.004 \pm 0.09$ when the DECIGO detected standard sirens and cosmic chronometers were used. When the standard siren (ET) is considered, the cosmic curvature estimate is $\Omega_{k}=0.009 \pm 0.13$. In other words the expected precision is $\Delta \Omega_{k}=10^{-2}$ with the DECIGO+CC and $\Delta \Omega_{k}=10^{-1}$ with the ET+CC. Compared to the latest model-independent estimations of the spatial curvature using the distance sum rule method [15], our results suggest a considerable improvement in precision when the future $\mathrm{GW}$ observatories provide rich statistics of $D_{L}(z)$ measurements. In order to surpass the limitations of small CC sample we reconstructed $H(z)$ function using GP technique and used it together with reconstructed $D_{L}(z)$ and $D_{L}^{\prime}(z)$ to investigate $\Omega_{k}(z)$. This is important to look if the current data, even though scarce, yet contain hints that cosmic curvature could locally deviate from the overall curvature assumed in FLRW metric. Such possibilities could be expected as a result of back-reaction of inhomogeneities, which entered non-linear regime during the structure formation [72].

Summarizing, the newly emerged gravitational wave astronomy can acquire another dimension of being useful in local measurements of cosmic curvature using distant sources. Such approach, essentially different from doing fits of globally defined (at the level of FLRW metric) curvature parameter. Possible deviations between these two approaches might be an extremely useful hints of phenomena and processes overlooked in current cosmological studies.

Acknowledgements We would like to thank Jingzhao Qi and Sixuan Zhang for their helpful discussions. This work was supported by National Key R\&D Program of China No. 2017YFA0402600; the National Natural Science Foundation of China under Grant Nos. 12021003, 11690023, 11633001 and 11920101003; Beijing Talents Fund of Organization Department of Beijing Municipal Committee of the CPC; the Strategic Priority Research Program of the Chinese Academy of Sciences, Grant No. XDB23000000; the Interdiscipline Research Funds of Beijing Normal University. J.-Z. Qi was supported by China Postdoctoral Science Foundation under Grant No. 2017M620661, and the Fundamental Research Funds for the Central Universities N180503014. M.B. was supported by the Foreign Talent Introducing Project and Special Fund Support of Foreign Knowledge Introducing Project in China. He was supported by the Key Foreign Expert Program for the Central Universities No. X2018002.

Data Availability Statement This manuscript has associated data in a data repository. [Authors' comment: The observational Hubble parameter data are available from the references cited in the paper, while the simulated luminosity distance data from DECIGO and ET are available from the corresponding author upon request.]

Open Access This article is licensed under a Creative Commons Attribution 4.0 International License, which permits use, sharing, adaptation, distribution and reproduction in any medium or format, as long as you give appropriate credit to the original author(s) and the source, provide a link to the Creative Commons licence, and indicate if changes were made. The images or other third party material in this article are included in the article's Creative Commons licence, unless indicated otherwise in a credit line to the material. If material is not included in the article's Creative Commons licence and your intended use is not permitted by statutory regulation or exceeds the permitted use, you will need to obtain permission directly from the copyright holder. To view a copy of this licence, visit http://creativecomm ons.org/licenses/by/4.0/.

Funded by $\mathrm{SCOAP}^{3}$.

\section{References}

1. S. Cao, J.Z. Qi, Z.J. Cao et al., NatSR 9, 11608 (2019)

2. J.Z. Qi, S. Cao, Y. Pan, J. Li, PRD 99, 063507 (2019)

3. D.H. Weinberg, M.J. Mortonson, D.J. Eisenstein et al., Phys. Rep. 530, 87 (2013)

4. N. Aghanim et al., Planck Collaboration. A\&A 641, A6 (2020)

5. K. Ichikawa, M. Kawasaki, T. Sekiguchi, T. Takahashi, JCAP 12, 005 (2006)

6. C. Clarkson, M. Cortes, B. Bassett, JCAP 08, 011 (2007)

7. Y.G. Gong, A. Wang, PRD 75, 043520 (2007)

8. J.M. Virey, D. Talon-Esmieu, A. Ealet, P. Taxil, A. Tilquin, JCAP 12, 008 (2008)

9. Z.X. Li, X.H. Ding, G.J. Wang, K. Liao, Z.H. Zhu, ApJ 854, 146 (2018)

10. S. Cao, J.Z. Qi, M. Biesiada et al., PDU 24, 100274 (2019)

11. E. Di Valentino, A. Melchiorri, J. Silk, Nat. Astron. 4, 196 (2020)

12. G. Bernstein, ApJ 637, 598 (2006)

13. S. Räsänen, K. Bolejko, A. Finoguenov, PRL 115, 101301 (2015)

14. J.Z. Qi, S. Cao, S.X. Zhang et al., MNRAS 483, 1104 (2019)

15. H. Zhou, Z.X. Li, ApJ 889, 186 (2020)

16. S. Cao, M. Biesiada, X.G. Zheng, Z.H. Zhu, ApJ 806, 66 (2015)

17. Y. Chen, R. Li, Y. Shu, MNRAS 488, 2977 (2019)

18. S. Cao, M. Biesiada, J. Jackson et al., JCAP 02, 012 (2017)

19. A. Shafieloo, C. Clarkson, PRD 81, 083537 (2010)

20. E. Mortsell, J. Onsson, arXiv:1102.4485 (2011)

21. D. Sapone, E. Majerotto, S. Nesseris, PRD 90, 023012 (2014)

22. R. Cai, Z. Guo, T. Yang, PRD 93, 043517 (2016)

23. B. Wang, J.Z. Qi, J.F. Zhang, X. Zhang, ApJ 898, 100 (2019)

24. Z.X. Li, G.J. Wang, K. Liao, Z.H. Zhu, ApJ 833, 240 (2016)

25. X.G. Zheng, X.H. Ding, M. Biesiada, S. Cao, Z.H. Zhu, ApJ 825, 17 (2016)

26. J.J. Wei, X.F. Wu, ApJ 838, 160 (2017)

27. G.J. Wang, J.J. Wei. Z.X. Li, J.Q. Xia, Z.H. Zhu, ApJ 847, 45 (2017)

28. A. Rana, D. Jain, S. Mahajan, A. Mukherjee, JCAP 03, 028 (2017)

29. G. Risaliti, E. Lusso, Nat. Astron. 3, 272 (2018)

30. F. Melia, MNRAS 489, 517 (2019)

31. J.J. Wei, F. Melia, ApJ 888, 99 (2020)

32. B.F. Schutz, Nature 323, 310 (1986)

33. J. Jiménez, JFM 842, P1 (2018)

34. J.J. Wei, ApJ 868, 29 (2018)

35. K. Liao, PRD 99, 083514 (2019)

36. M. Seikel, C. Clarkson, M. Smith, JCAP 6, 036 (2012)

37. T. Yang, Z.K. Guo, R.G. Cai, PRD 91, 123533 (2015)

38. H. Yu, F.Y. Wang, ApJ 828, 85 (2016)

39. T.H. Liu, S. Cao, J. Zhang et al., ApJ 886, 94 (2019)

40. Y. Wu, S. Cao, J. Zhang et al., ApJ 888, 113 (2020)

41. X.G. Zheng, K. Liao, M. Biesiada et al., ApJ 892, 103 (2020)

42. D.W. Hogg, arXiv:astro-ph/9905116 (1999)

43. S. Kawamura, M. Ando, N. Seto et al., CGQ 28, 094011 (2011)

44. N. Seto, S. Kawamura, T. Nakamura, PRL 87, 221103 (2001)

45. S. Kawamura, Decigo Working Group. qopu.conf (2019)

46. S. Kawamura. T. Nakamura, M. Ando et al., IJMPD 28, 1845001 (2019)

47. B.P. Abbott et al., LIGO Scientific Collaboration and Virgo Collaboration. PRL 116, 061102 (2016) 
48. B.P. Abbott et al., LIGO Scientific Collaboration and Virgo Collaboration. PRL 119, 161101 (2017)

49. L.E. Kidder, C.M. Will, A.G. Wiseman, PRD 47, 3281 (1993)

50. C. Cutler, E.E. Flanagan, PRD 49, 2658 (1994)

51. S. Kawamura et al., CQG 23, 125 (2006)

52. R. Cai, T. Yang, PRD 95, 044024 (2017)

53. W. Zhao, C.V.D. Broeck, D. Baskaran, T.G.F. Li, PRD 83, 023005 (2011)

54. B. Sathyaprakash et al., CQG 27, 215006 (2010)

55. R. Schneider et al., MNRAS 324, 797 (2001)

56. C. Cutler, D.E. Holz, PRD 80, 104009 (2009)

57. The Einstein Telescope Project, http://www.et-gw.eu/index.php/ etdsdocument

58. J.Z. Qi, S. Cao, M. Biesiada et al., PRD 100, 023530 (2019)

59. J.Z. Qi, S. Cao, Y. Pan, J. Li, PDU 26, 100338 (2019)

60. D. Foreman-Mackey, D.W. Hogg, D. Lang, J. Goodman, PASP 125, 306 (2013)

61. R. Jimenez, A. Loeb, ApJ 573, 37 (2002)
62. E. Gaztä̈aga, A. Cabre, L. Hui, MNRAS 399, 1663 (2009)

63. C. Blake, S. Brough, M. Colless et al., MNRAS 425, 405 (2012)

64. L. Samushia, B.A. Reid, M. White et al., MNRAS 429, 1514 (2013)

65. A. Font-Ribera, D. Kirkby, N. Busca et al., JCAP 5, 027 (2014)

66. T. Delubac, J.E. Bautista et al., A\&A 574, A59 (2015)

67. A. Shafieloo, A.G. Kim, E.V. Linder, PRD 85, 123530 (2012)

68. M. Seikel, C. Clarkson, arXiv:1311.6678 (2013)

69. S. Yahya, M. Seikel, C. Clarkson et al., PRD 89, 023503 (2014)

70. A. Banerjee, E. Ó Colgáin, M. Sasaki, M. M. Sheikh-Jabbari, T. Yang, arXiv:2009.04109 (2020)

71. T. Yang, A. Banerjee, E. Ó Colgáin, arXiv:1911.01681

72. K. Bolejko, CQG 35, 024003 (2017) 